\title{
Über die Wirkung einer Abrundung auf die Torsionsspannungen in der inneren Ecke eines Winkeleisens.
}

\author{
Von E. TREFFTZ in Dresden.
}

D ie in der technischen Elastizitätslebre regelmäßig gestellte Frage nach der größten, in einem Bauteil auftretenden Spannung muß zuweilen dahin beantwortet werden, daß sich rechnerisch unendlioh groBe Spannungen ergeben, wie z. B. bei der Torsion prismatischer Stäbe an einspringenden Ecken des Querschnitts. Die Frage bedarf dann elner Modifikation; wir mïssen untersuchen, welche Abrundang einer solchen einspringenden Eoke oder Kante die Spannung auf das zulässige Maß reduziert, bezw. wie weit eine Spannungserniedrigung durch eine Abrundung überhaupt zu erreichen ist.

In der vorliegenden Arbeit berechne ich den Einfluß einer Abrundung auf die Torsionsspannungen in der einspringenden Ecke eines Winkeleisens, und zwar entwiokle ich ein Näherungsverfahren für schwache Abrundungen (klein $\Theta$ Abrundnngsbalbmesser). Eine Ergänzung hierzu wird demnächst folgen; in einer Dissertation werden einige spezielle Fälle stärkerer Abrundung behandelt werden.

\section{Die Aufgabe und das Prinzip der Näherungs-}

lösung. Wir betrachten ein Winkeleisen, dessen Schenkel gleiche Breite $b=1$ haben, und nehmen, was für unsere Fragestellang zulässig ist, an, daß sie sich ins Unendliche erstrecken. In bekannter Weise kann man den Spannungszustand durch eine bydrodynamische Analogie deuten ${ }^{1}$ ). Sind $u$ und $v$ die Geschwindigkeitskomponenten der Strömung einer inkompressiblen Flüssigkeit, deren Rotation konstant und zwar gleich 2 ist, and für die der Rand des Querschnitts Stromlinie ist, so lassen sich die Komponenten der Schnbspannung tür die $x$ - und $y$-Richtung in der Form darstellen:

$$
\tau_{\iota x}=G \omega u, \tau_{y z}=G \omega v .
$$

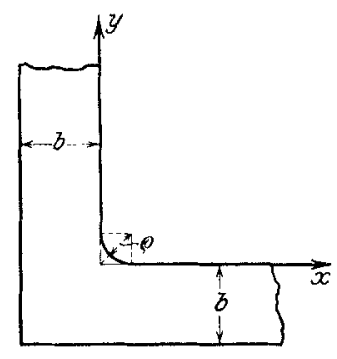

Abb. 1

wo $G$ den Schnbmodul, $\infty$ die Verdrehung pro Längeneinheit des gedrillten Stabes bedeutet. Das Koordinatensystem ist dabei so gelegt, dab die $z$ Achse mit der Richtung der Stabachse zusammenfällt und der Nullpunkt in die einspringende Ecke fällt. Beim Umströmen der einspringenden Ecke wird nun die Geschwindigkeit unendlich, und demnach ergeben sich hier auch unendliche Schubspannungen. Ueber den Charakter dieser Singalaritat gewinnen wir folgendermaßen AufschluB. Wir stellen uns vor, daB wir der genannten Strömung von konstantem Wirbel in der Umgebung der Ecke eine wirbelfreie Strömung ïberlagern, die der ersten entgegengerichtet ist. Wenn wir die Stärke dieser Strömung passend bestimmen, so wiird an der Ecke gerade die Geschwindigkeit Null herauskommen, $d$. h. im Spannungsproblem die Spannung Null. Das bedeutet, daB wir die Strömung, die uns die Schubspannungen liefert, in der Nähe der Ecke zerlegen können in eine Strömung von konstantem Wirbel, die an der Ecke die Geschwindigkeit Null hat, und in eine Potentialströmung, die das Unendlichwerden der Spannung darstellt. Nun ist das komplexe Potential einer eine scharfe Ecke umströmenden wirbelfreien Strömung

$$
F(z)=C z^{2 / 3} \text {. . . . . . . . . . . . . (2), }
$$

dessen Ableitung die Geschwindigkeitskomponenten liefert:

$$
u-i v=F^{\prime \prime}(z)={ }^{2 / 3} C_{z}-1 / 8
$$

Die Konstante $C$ ist die charakteristische Konstante der Singularität, sie muB aus der Lösung des Spannungsproblems für das nicht abgerundete Winkeleisen "entnommen werden. In einer Arbeit in den mathematischen Annalen ${ }^{2}$ ) habe ich diese Lösung für das Winkeleisen mit unendlich langen Schenkeln gegeben; aus der numerischen Durch-

1) Vergl. den Zus. Bericht von Th. Pöschl, diese Zeitschr. 1, 1921, S. 314.

2) E. Trefft $z$, Ueber die Torsion prismatischer Stabe von polygonalem Querschnitt. Math. Ann. Band 82,1920 , Heft $1 / 2$, Seite 97 bis 112 . 
rechnung ergibt sich $C$ zu 1,48. In der Umgebung der Ecke werden also die Spannungskomponenten

$$
\tau_{\prime \prime z}-i \tau_{y z}=\frac{0,988}{\sqrt[3]{3}} G \omega
$$

Wird jetzt die einspringende Ecke abgerundet, so fragt es sich, wie grob die Maximalspannung in dem innersten Punkte wird. Der strengen Lösung steht hier die Schwierigkeit der numerischen Durchführung im Wege. Wir wollen deshalb die folgende Näherungsmethode anwenden. Den Teil der Spannungen, welcher der Strömung mit konstantem Wirbel entspricht, wollen wir vernachlässigen, denn er wird in der Ecke Null und kommt somit gegen den dort unendlich werdenden Teil (4), der der Potentialströmang entspricht, nicht in Frage. Bezïglich dieses letzteren Teils bedenken wir, daß sich die Wirkung der Abrundung schon in geringer Entfernung nicht mehr bemerkbar macht. Wir werden also eine Potentialströmung suchen, die um die abgerundete Ecke strömt und sich in einiger Entfernung von der Ecke, d. h. in mathematischer Idealisierung: im Unendlichen verhält wie die Strömung um die unabgerundete Ecke. Wir erhalten diese Strömung, indem wir das von der abgerundeten Ecke begrenzte Gebiet, und zwar das naoh der konvexen Seite gelegene, auf die Halbebene abbilden; dabei gehen die Stromlinien in die Geraden $\eta=$ konst. über (siehe Abb. 2 c).

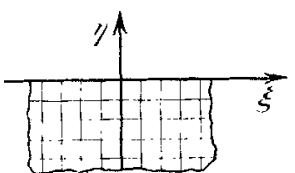

Abb. $2 \mathrm{a}$

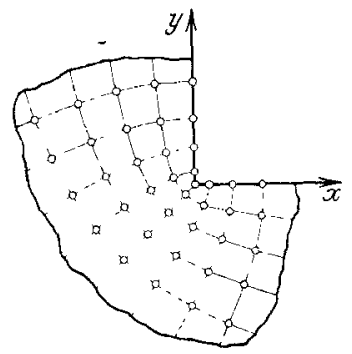

Abb. 2 b

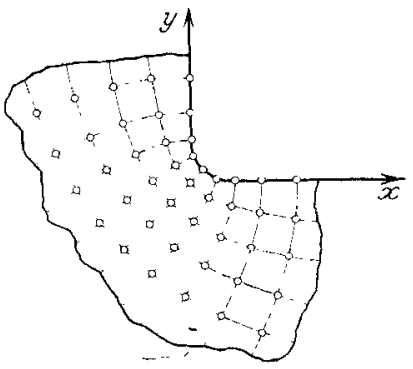

Abb. $2 \mathrm{c}$

2. Die Abbildung der abgerundeten Ecke auf die Halbebene. Zur Abbildung der abgerundeten Ecke auf die Halbebene stehen uns die Methoden von Schwarz und Christoffel zur Verfügung, die wir am einfachsten folgendermaken darstellen.

Es sei

$$
z=f(\zeta)
$$

die Abbildungsfanktion, die die untere Halbebene der 5 -Ebene in das von der abgerundeten Ecke begrenzte Gebiet überfïhrt. Die Ableitung dieser Funktion bezeichnen wir mit $z^{\prime}=p+i q$, es ist also

$$
p=\frac{\partial x}{\partial \xi}=\frac{\partial y}{\partial \eta}, q=-\frac{\partial x}{\partial \eta}=\frac{\partial y}{\partial \xi} .
$$

die zweite Ableitung sei $z^{\prime \prime}=s+i t$, es ist also

$s=\frac{\partial \boldsymbol{p}}{\partial \xi}=\frac{\partial q}{\partial \eta}=\frac{\partial^{2} x}{\partial \xi^{2}}=\frac{\partial^{2} y}{\partial \xi \partial \eta}=-\frac{\partial x}{\partial \eta^{2}}, \quad t=-\frac{\partial \boldsymbol{p}}{\partial \eta}=\frac{\partial q}{\partial \xi}=-\frac{\partial^{2} x}{\partial \xi \xi \eta}=-\frac{\partial^{2} y}{\partial \eta^{2}}=\frac{\partial^{2} y}{\partial \xi^{2}}$

Betrachten wir nun irgend einen Punkt der $\xi$-Aohse, so erhalten wir aus (5) mit $\eta=0$ die Randpunkte in der $z$ Ebene

$$
z=f(\xi), x=x(\xi), y=y(\xi) .
$$

Die Koordinaten $x$ und $y$ sind hier als Funktionen des Parameters $\xi$ dargestellt; es ist also die Krümmung des Randes in der $z$-Ebene

$$
k=\frac{\frac{\partial x}{\partial \xi} \frac{\partial^{2} y}{\partial \xi^{2}}-\frac{\partial y}{\partial \xi} \partial^{2} x}{\sqrt{\left(\begin{array}{c}
\partial x \\
\partial \xi
\end{array}\right)^{2}+\left(\begin{array}{l}
\partial y \\
\partial \xi
\end{array}\right)^{2}}}=\frac{p t-q s}{\left(p^{2}+q^{2}\right)^{8 / 2}}
$$

Bezeichnen wir mit $J(f)$ den Imaginärteil einer Funktion $f$, mit $k$ den Realteil, so wird nach (6) und (7)

$$
k=\frac{1}{\left|z^{\prime}\right|} J^{z^{\prime \prime}}
$$


oder:

$$
k \sqrt{p^{2}+q^{2}}=J\left(\frac{z^{\prime \prime}}{z^{\prime}}\right)
$$

Nun ist längs der einzelnen Begrenzungsstücke $k$ konstant; gehen wir also längs der $\xi$-Achse weiter, so erhalten wir durch Differentiation nach $\xi$

Nun ist für $d \zeta=d \xi=$ reell

$$
k \frac{p s+q t}{\sqrt{p^{2}+q^{2}}}=\frac{\partial}{\partial \xi} J\left(\frac{z^{\prime \prime}}{z^{\prime}}\right)
$$

$$
\frac{\partial}{\partial \xi} J\left(\frac{z^{\prime \prime}}{z^{\prime}}\right)=J \frac{d}{d \xi}\left(\frac{z^{\prime \prime}}{z^{\prime}}\right)
$$

Die rechte Seite wird also einfach

$$
\begin{gathered}
J \frac{d}{d \zeta}\left(\frac{z^{\prime \prime}}{z^{\prime}}\right) . \\
k V^{\prime} \frac{q^{\prime \prime}}{p^{2}+q^{2}} R\left(\frac{z^{\prime \prime}}{z^{\prime}}\right) \\
J\left(\frac{z^{\prime \prime}}{z^{\prime}}\right) R\left(\frac{z^{\prime \prime}}{z^{\prime}}\right) \\
=\frac{1}{2} J\left(\frac{z^{\prime \prime}}{z^{\prime}}\right)^{2} .
\end{gathered}
$$

Die linke Seite wird

oder bei Benutzung von (10):

Wir erhalten somit die Bedingung dafür, daß die Krümmung konstant ist,

$$
J\left\{\frac{d}{d \zeta}\left(\frac{z^{\prime \prime}}{z^{\prime}}\right)-\frac{1}{2}\left(\frac{z^{\prime \prime}}{z^{\prime}}\right)^{2}\right\}=0
$$

Wäre der Ausdruck $\frac{d}{d \zeta}\left(\frac{z^{\prime \prime}}{z^{\prime}}\right)-\frac{1}{2}\left(\frac{z^{\prime \prime}}{z^{\prime}}\right)^{2}$ überall regulär, so könnten wir aus dem Verschwinden seines Imaginärteils längs der reellen Achse schließen, daB er überall Null wäre. Nun sollen den Punkten $\zeta=-1$ und $\zeta=+1$ die Punkte in der $z$-Ebene entsprechen, an denen der Kreisbogen an die Geraden anschließt; in diesen Punkten hat $z=f(\zeta)$ eine Singularitat, also auch der Ausdruck (12).

Den Charakter dieser Singularität erkennen wir daraus, daf an diesen Punkten die Krümmung sich sprungweise ändert; es muß also $(10)$ der Imaginärteil von $\left(\frac{z^{\prime \prime}}{z^{\prime}}\right)$ eine Singularitat haben wie der Imaginärteil von $c \ln (\xi \pm 1)$. Wir erfüllen das, indem wir für $\frac{d}{d \zeta}\left(\frac{z^{\prime \prime}}{z^{\prime}}\right)-\frac{1}{2}\left(\frac{z^{\prime \prime}}{z^{\prime}}\right)^{2}$ in den Punkten $\zeta=-1$ and $\zeta=+1$ einfache Pole annehmen. Schließlich haben wir im Unendlichen $z=\zeta^{3 / 2}$, also

$$
\frac{d}{d \zeta}\left(\frac{z^{\prime \prime}}{z^{\prime}}\right)-\frac{1}{2}\left(\frac{z^{\prime \prime}}{z^{\prime}}\right)^{2}=-\frac{5}{8} \zeta^{-2}
$$

Im Unendlichen muß also $\frac{d}{d \zeta}\left(\frac{z^{\prime \prime}}{z^{\prime}}\right)-\frac{1}{2}\left(\frac{z^{\prime \prime}}{z^{\prime}}\right)^{2}$ wie $-\frac{5}{8} \frac{1}{\zeta^{2}}$ gegen Null gehen. Setzen wir

$$
\frac{d}{d \zeta}\left(\frac{z^{\prime \prime}}{z^{\prime}}\right)-\frac{1}{2}\left(\frac{z^{\prime \prime}}{z^{\prime}}\right)^{2}=-\frac{5}{8} \frac{1}{\zeta^{2}-1}
$$

so sind die Bedingungen für $\zeta= \pm 1$ und im Unendlichen erfüllt; ebenso gilt (12), so daß die Konstanz der Krümmung längs der einzelnen Begrenzangsstücke gesichert ist. In (13) haben wir die Differentialgleichung gefunden, der unsere Abbildungsfunktion genügt. Wir verwandeln sie in eine lineare Differentialgleichnng, wenn wir $w=\frac{1}{\sqrt{z^{\prime}}}$ als neue Variable einführen. Gl. (13) wird dann:

$$
w^{\prime \prime}-\frac{5 w}{16\left(\zeta^{2}-1\right)}=0
$$

In dieser Form verwenden wir die Gleichung zur Integration; wir entwiokeln die Lösung einmal im Unendlichen, dann in der Umgebung von $\zeta=0$ und bestimmen die Integrationskonstanten der Entwicklung am Nullpunkt so, dab sich diese Entwicklung in den Punkten $\zeta=-1$ und $\zeta=+1$ an die Entwicklung um $\zeta=\infty$ anschließt. Die erste Entwioklung konvergiert außerhalb, die zweite innerhalb des Einheitskreises, so daf wir damit die ganze Abbildung beherrschen.

Setzen wir also im Unendlichen (unter Berücksichtigung des Charakters von $z=f(\zeta)$ im Unendlichen)

$$
w=C_{1} \zeta^{-1 / 4}+C_{5} \zeta^{-5 / 4}+C_{9} \zeta^{-\% / 4}+\ldots
$$


so erhalten wir für die Koeffizienten aus der Differentialgleichnng die Rekursionsformeln:

$$
C_{8 n+9}=\frac{(8 n+1)(8 n+5)}{(8 n+9)(8 n+13)-5} C_{8 n+1}
$$

wo $C_{1}$ als Integrationskonstante stehen bleibt, und $C_{5}, C_{13}, C_{21}$ usw. Null werden. Numerisch ergeben sich daraus die Werte:

$C_{9}=0,0446 C_{1}, C_{17}=0,0148 C_{1}, C_{25}=0,0073 C_{1}, C_{33}=0,0044 C_{1}, C_{41}=0,0039 C_{1}$ (17).

Setzt man diese ein nnd schätzt den Rest nach elementaren Methoden ab, so erhält man für

$$
\zeta= \pm 1 w(1)=C_{1}(1,092 \pm 0,004), w(-1)=C_{1} e^{i \pi / 4}(1,092 \pm 0,004)
$$

Für die Entwicklung am Nulpunkte setzen wir an:

$$
w=\gamma_{0}+\gamma_{1} \zeta+\gamma_{2} \zeta^{2}+\gamma_{3} \zeta^{3}+
$$

nnd erhalten für die Koeffizienten die Rekursionsformeln

$$
\gamma_{\nu+2}=\frac{\nu(\nu-1)-5 / 16}{(\nu+2)(\nu+1)} \gamma_{\nu}
$$

$\gamma_{0}$ und $\gamma_{1}$ bleiben als Integrationskonstanten unbestimmt, für die übrigen ergibt die numerische Auswertung:

$$
\left.\begin{array}{l}
\gamma_{2}=-0,1563 \gamma_{0}, \quad \gamma_{3}=-0,0521 \gamma_{1} \\
\gamma_{4}=-0,0220 \gamma_{0}, \quad \gamma_{5}=-0,0148 \gamma_{1} \\
\gamma_{6}=-0,0086 \gamma_{0}, \quad \gamma_{7}=-0,0069 \gamma_{1} \\
\gamma_{5}=-0,0045 \gamma_{0}, \quad \gamma_{9}=-0,0040 \gamma_{1}
\end{array}\right\}
$$

Setzt man diese Werte ein und schätzt wieder den"Rest ab, so ergibt sich mit einer Genauigkeit von $1 / 2 \mathrm{vH}$

$$
\left.\begin{array}{rl}
w(1) & =0,791 \gamma_{0}+0,906 \gamma_{1} \\
w(-1) & =0,791 \gamma_{0}-0,906 \gamma_{1}
\end{array}\right\} \quad . \quad . \quad . \quad \cdot \quad \cdot \quad \text { (22). }
$$

Diese Werte müssen den oben ermittelten gleich sein, d. h. es ist:

$$
\begin{aligned}
& 0,791 \gamma_{0}+0,906 \gamma_{1}=1,092 C_{1}, \\
& 0,791 \gamma_{0}-0,906 \gamma_{1}=1,092 e^{i \pi / 4} C_{1},
\end{aligned}
$$

woraus sich $\gamma_{0}$ und $\gamma_{1}$ berechnen:

$$
\gamma_{0}=0,690\left(1+e^{i \pi / 4}\right) C_{1}, \gamma_{1}=0,603\left(1-e^{i \pi / 4}\right) C_{1}
$$

Die übrig bleibende Integrationskonstante $C_{1}$ bestimmt sich aus der Krümmung des abrundenden Kreisbogens unserer Ecke. An einem beliebigen Randpunkte ist die Krümmung $k=\frac{1}{\left|z^{\prime}\right|} J\left(\frac{z^{\prime \prime}}{z^{\prime}}\right)$ oder, in $w=\frac{1}{\sqrt{z^{\prime}}}$ ansgedrückt:

$$
k=-2|w|^{2} J\left(\frac{w^{\prime}}{w}\right) \text {. }
$$

Setzen wir hier die Werte von $w$ und $w^{\prime}$ für $\zeta=0$ ein, $w(0)=\gamma_{0}, w^{\prime}(0)=\gamma_{1}$, so ergibt sich $k_{0}=-2 \gamma_{0} \overline{\gamma_{0}} J\left(\frac{\gamma_{1}}{\gamma_{0}}\right)$ oder

\section{Daraus ergibt (23)}

$$
\begin{gathered}
k_{0}=-2 J\left(\overrightarrow{\gamma_{0} \gamma_{1}}\right) . \\
k_{0}=1,18 \bar{C}_{1}^{2} .
\end{gathered}
$$

und damit ist unsere Aufgabe soweit gelöst, als sie für unsere Aufgabe von Bedeutung ist. Auf die Aufstellung der Reihen für $z$ können wir verzichten, da für die Spannungsberechnung nur $z^{\prime}=\frac{1}{w^{2}}$ in Frage kommt und wir dieses auch nur im innersten Punkte des Kreisbogens brauchen, d. h. fïr $\zeta=0$, well uns ja nur dio Maximalspannung interessiert.

3. Die maximale Spannung in der einspringenden Ecke. Nach Abschnitt 1 erhalten wir die Spannungskomponenten in der Umgebung der Eoke in der Form:

$$
\tau_{x z}-i \tau_{y z}=G \omega F^{\prime}(z)
$$

aus der Ableitung des komp?exen Potentials $F(z)$ für eine die Ecke umströmende Poten. tialströmung, dessen Ableitung im Unendlichen von der Form $F^{\prime}(z)=\frac{0.988 \theta \dot{\omega}}{\sqrt[3]{z}}$ ist. Da in der $\zeta$-Ebene die Stromilinien einfach die Geraden $\eta=$ konst. sind, so wird das gesmchte komplexe Strömungspotential $F(z)=A \zeta$. 
die Spannungen werden also:

$$
\tau_{x z}-i \tau_{y z}=G \omega A \frac{d \zeta}{d z}=G \omega A w^{2}
$$

Die Konstante $A$ bestimmt sich aus der vorstehenden Bedingung für das Unendliche; im Unendlichen wird $\frac{d \zeta}{d z}=w^{2}=C_{1}^{2} \zeta-1 / 2$, daratıs folgt durch Integration $\zeta=\left(\frac{3 C_{1}^{2}}{2} z\right)^{2 / s}$, also

$$
\tau_{x z}-i \tau_{y z}=G \omega A C_{1}^{2}\left(\frac{3 C_{1}^{2}}{2} z\right)^{-1 / 3}=G \omega \sqrt{\frac{2}{3}} A C_{1}^{4 / 3} z^{-1 / 3} \text {. . . (29). }
$$

Die Uebereinstimmung mit (4) erfordert $\sqrt[3]{\frac{2}{3}} A C_{1}{ }^{4 / 3}=0,988$ oder $A=1.13 C_{1}^{-w^{-4}}$. Die größte Spannung tritt im innersten Punkt des abrundenden Kreisbogens auf, d. b. fuïr $\zeta=0$, hier wird

$$
\tau_{\max }=\sqrt{\tau_{x z}{ }^{2}+\tau_{y z}{ }^{2}}=G \omega A\left|w_{0}\right|^{2}=G \omega A \gamma_{0} \bar{\gamma}_{0} .
$$

Wir erhalten darans für die maximale Spannung

$$
\tau_{\max }=G \omega 1,13 C_{1}^{-4 / 3} 1,625 C_{1}^{2}=\frac{1,74 G \omega}{\sqrt[3]{\varrho}}
$$

Dies ist unser Hauptresultat, es gibt uns vor allen Dingen die Art des Unendlichwerdens von $\tau_{\max }$ mit abnehmendem Abrundungshalbmesser.

Es ist zweokmäBig, sioh von der Spezialisierung $b=1$ frei zu machen und das eben gewonnene Resultat noch in etwas veränderter Form darzustellen. Erstrecken sich die Schenkel des Winkeleisens ins Unendliche, so geht dis Spannung an dem Rande gegen den Wert $\tau_{\infty}=G a b$ und zwar năhert sioh sowobl an der Oberseite wie an der Unterseite $\tau$ diesem Werte sehr rasch (exponentiell). Auch für Winkeleisen endlioher Lünge untersoheidet sich die Maximalspannung an den Schenkeln nur wenig von diesem Werte. Mit Benutzung dieser Spannung schreibt sich (30)

$$
\frac{\tau_{\max }}{\tau_{\infty}}=1,74 \sqrt[3]{\frac{b}{Q}}
$$

wobei die Spezialisierung $b=1$ aufgegeben ist. Es lige natürlich nahe, die Abrundung so zu whhlen, daB $\tau_{\max }=\tau_{\infty}$ würde, doch wäre das schon eine so starke Abrundung, daB unsere Näherungsmethode nicht mehr anwendbar ware. An einigen Beispielen stärkerer Abrundung hat Hr. Dassen in einer Aachener Dissertation das Resultat erhalten, das fiir starke Abrundung ziemlioh unabhängig von dem Krümmungshalbmesser $\tau_{\max }=2 \tau_{\infty}$ gesetzt werden kann.

\title{
Spannungsverteilung in Blechen mit mehreren kreisrunden Löchern.
}

\author{
Von C. WEBER in Hildesheim.
}

$Z$ den ungelösten Aufgaben der Elastizitatslehre gehört die Frage nach der Spannungsverteilung in Blechen mit mehreren kreisrunden Löohern. Von Wichtigkeit ist besonders die Spannungserhöhnng am Rande der Löcher. In vorliegender Arbeit gebe ich die bei einer früheren Gelegenbeit ${ }^{1)}$ angekündigte Lösung für den Fall der allseitig oder in einer Richtnng gezogenen unendlichen Scheibe mit zwei Löchern. Am SohluB wird ein Zahlenbeispiel durchgerechnet und werden Schlubfolgerungen für die mehrlach durchlochte Sohelbe gezogen.

1. Aufsuchung einer Tefllösung. Die zwei verschleden großen Löcher, Abb. 1, gehören dem Systeme einer zweipoligen Kreisschar an, deren Polabstand gleloh der Lungeneinheit genommen ist. Durch die

J) Diese Zeltschr. 2, 1922, S. 185-187.

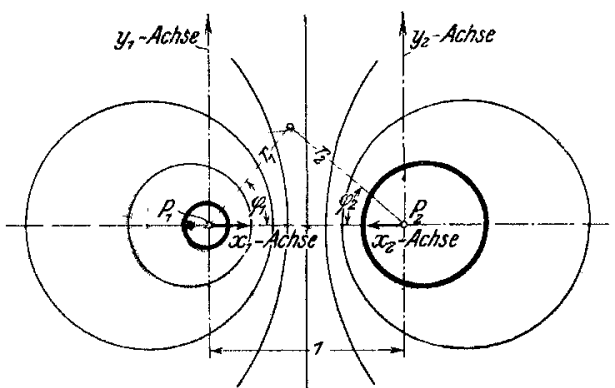

Abb. 1 